\title{
Mediterranean Diet and Essential Tremor
}

\author{
A Case-Control Study \\ Nikolaos Scarmeas ${ }^{a-c}$ Elan D. Louis ${ }^{b, c}$ \\ ${ }^{a}$ Taub Institute for Research in Alzheimer's Disease and the Aging Brain, ${ }^{\mathrm{b}}$ The Gertrude H. Sergievsky Center, and \\ 'Department of Neurology, Columbia University Medical Center, New York, N.Y., USA
}

\section{Key Words}

Essential tremor - Epidemiology $\cdot$ Mediterranean diet

\begin{abstract}
Background: Higher adherence to the Mediterranean diet (MeDi) has been related to lower Alzheimer's disease risk. Some dietary factors have been studied in patients with essential tremor (ET), but the MeDi's effect has not been investigated. Methods: Adherence to the MeDi was calculated from a food frequency questionnaire administered in a casecontrol study of environmental epidemiology of ET in the New York Tri-State area. Logistic regression models were used to examine whether adherence to the MeDi predicted ET (vs. control) outcome. The models adjusted for age, gender, ethnicity, education, caloric intake, body mass index, smoking, ethanol consumption, coffee intake and blood harmane concentrations. Results: 148 ET cases adhered less to MeDi (0-9 scale with higher scores indicating higher adherence) than 250 controls (mean $4.3 \pm 1.7$ vs. $4.7 \pm 1.7$; $\mathrm{p}=0.03$ ). Higher adherence to MeDi was associated with lower odds for ET [0.78 (0.61-0.99); $p=0.042]$. As compared to subjects at the lowest MeDi adherence tertile, those at the middle tertile had lower ET odds [0.41 (0.16-1.05)], while subjects at the highest tertile had an even lower ET odds [0.29 (0.10-0.82); $p$ for trend 0.021]. Conclusions: Compared to
\end{abstract}

RO1 NS 39422, P30 ES09089, RR00645 from the National Institutes of Health, Bethesda, Md., USA.

\section{KARGER \\ Fax +41 613061234 \\ E-Mail karger@karger.ch}

(c) 2007 S. Karger AG, Basel

www.karger.com
Accessible online at:

www.karger.com/ned controls, ET cases adhered less to MeDi. The gradual reduction in ET odds with higher MeDi adherence tertiles suggests a possible dose-response effect. The mechanisms that underlie this association merit further study.

Copyright $\odot 2007$ S. Karger AG, Basel

\section{Introduction}

A variety of dietary factors have been studied in patients with late-life neurological disorders, including Parkinson's disease, Alzheimer's disease and, more recently, essential tremor (ET). Individuals do not consume foods or nutrients in isolation but rather as components of their daily diet, yet most previous research regarding biological effects of nutrition has focused on individual food categories or nutrients. Dietary pattern analysis can integrate complex or subtle interactive effects of many dietary constituents. It bypasses the problems generated by multiple testing and by high correlations that can exist among dietary constituents [1]. Therefore, dietary patterns have the ability to capture the diet's multidimensionality while reducing its apparent complexity.

There are growing data regarding the effect of composite dietary patterns on neurological diseases. In two recent studies, higher adherence to the Mediterranean diet $(\mathrm{MeDi})$ was associated with lower risk for Alzheimer's disease [2, 3]. The MeDi consists of: (1) high intake of vegetables, legumes, fruits, cereals, fish, monounsaturated fatty acids, (2) low intake of saturated fatty acids,

Elan D. Louis

Unit 198, Neurological Institute

710 West 168 th Street

New York, NY 10032 (USA)

Tel. +1 212305 9194, Fax +1 212305 1304, E-Mail ed12@columbia.edu 
dairy products, meat and poultry, and (3) mild to moderate ethanol use. The MeDi may exert its beneficial health effects via multiple potential means; these include vascular, inflammatory and oxidative mechanisms [2].

The etiology of ET is poorly understood [4], and a better understanding of disease etiology and mechanisms has the potential to modify or prevent a disorder that affects as much as $4 \%$ of the adult population $[5,6]$. In addition to genetic factors, nongenetic factors (e.g., toxins, dietary factors) likely play a role in the etiology of this disease as well [4]. A variety of toxic [7-10] and dietary factors $[7,11,12]$ have been examined in ET. It has recently been suggested that one of the possible mechanisms for the pathogenesis of ET is neurodegeneration [13-15]. Thus, it is reasonable to assume that environmental or dietary factors that play a role in other neurodegenerative diseases (i.e. MeDi in Alzheimer's disease) could be important for ET as well.

Given the above, we wanted to further investigate the potential contribution of dietary factors in ET. More specifically, we examined the association between MeDi and ET status using data from a case control study of environmental risk factors for ET $[8,9]$. We hypothesized that higher adherence to the MeDi would be associated with lower odds for ET.

\section{Methods}

\section{Participants}

As documented previously, all participants were enrolled in a study of the environmental epidemiology of ET [8, 9]. Participants were all patients with ET at the Neurological Institute of New York, Columbia University Medical Center (CUMC), where they had received a diagnosis of ET from their treating neurologist. Patients were identified from computerized billing databases at CUMC. All such identified ET patients were selected for further office record review and then possible enrollment. Office records were reviewed (E.D.L.), and patients with diagnoses or physical signs of dystonia, Parkinson's disease, or spinocerebellar ataxia were excluded.

Controls were identified from the New York Tri-State area using random digit telephone dialing. Patients and controls were ascertained from the same source population in the New York Tri-State region (i.e., controls were selected from the same set of zip codes in New York, N.J., and Connecticut as were the patients with ET). The majority of these controls also received their health care at the same medical center as did the patients with ET (CUMC). These controls were frequency-matched to CUMC patients on 5-year age strata, gender, and ethnicity. Control recruitment proceeded more rapidly than patient recruitment, resulting in a larger number of controls than patients.

This study was approved by Columbia University Medical Center's Institutional Review board and it has been performed in accordance with the ethical standards of the 1964 Declaration of Helsinki. All subjects of this study gave their written informed consent prior to their participation.

To date, 461 subjects have been enrolled. Of these 461, 398 (86\%, 148 patients with definite or probable ET and 250 controls) were included in these analyses. The remaining 63 were excluded because they had a diagnosis of possible ET, they had other coexisting neurological conditions (e.g., Parkinson's disease or dystonia), or they failed to meet diagnostic criteria for ET. The 63 who were excluded did not differ from the 398 who were included in terms of gender or ethnicity, but they were slightly more educated (16.1 vs. 14.9 years of schooling; $\mathrm{p}=0.016$ ) and approximately 11 years younger $(57.8$ vs. 68.6 years; $\mathrm{p}<0.001)$.

\section{Clinical Evaluation}

All subjects were evaluated in person by a trained tester who collected demographic, medical, and family history information. The tester videotaped a tremor examination in all participants $[18,19]$. The tremor examination included assessments of postural, kinetic, intentional, and rest tremors. The videotaped examination also included an assessment of parkinsonian features (speech, facial expression, rapid alternating movements, writing, posture, and gait). Based on these data, the diagnosis of probable or definite ET was confirmed by the rater (E.D.L.) using published diagnostic criteria (moderate amplitude or greater tremor during four or more activities or a head tremor in the absence of Parkinson's disease, dystonia or another neurological disorder) $[18,19]$. None of the patients or control subjects had Parkinson's disease or dystonia.

\section{Predictors}

Diet

Dietary data regarding average food consumption over the past year were obtained using a 61-item version of Willett's Semiquantitative Food Frequency Questionnaire (Channing Laboratory, Cambridge, Mass., USA) [20]. Trained interviewers administered the questionnaire in English or Spanish. It has shown good reliability and validity related to recent nutrient intake [20].

Similarly to our previous work $[2,3]$, we followed the method described by Trichopoulou et al. [21] for the construction of the $\mathrm{MeDi}$ score. More specifically, we first regressed caloric intake (kcal) and calculated the derived residuals of daily gram intake - as recommended by Willett and Stampfer [22] - for each of the following seven categories [which define the components of the $\mathrm{MeDi}$ as previously defined in ref. 21]: dairy, meat, fruits, vegetables, legumes, cereals and fish. A value of 0 or 1 was assigned to each of the seven above groups, using sex-specific medians as cutoffs. For beneficial components (fruits, vegetables, legumes, cereals and fish) persons whose consumption was below the median were assigned a value of 0 , and persons whose consumption was at or above the median were assigned a value of 1 . For components presumed to be detrimental (meat and dairy products) persons whose consumption was below the median were assigned a value of 1 , and persons whose consumption was at or above the median were assigned a value of 0 . For fat intake (eighth food category) we used the ratio of daily consumption (in grams) of monounsaturated lipids to saturated lipids [21] (again using sex-specific median cutoffs for assignment values of 0 for low and 1 for high). For ethanol intake (ninth food category), subjects were assigned a score of 0 for either no $(0 \mathrm{~g} /$ day $)$ or more than moderate 
( $30 \mathrm{~g} /$ day) consumption, and a value of 1 for mild-moderate ethanol consumption ( $>0$ to $<30 \mathrm{~g} /$ day). This is in agreement with previous reports [21], that consider moderate amount of ethanol consumption as another characteristic component of the MeDi. The MeDi score was generated for each participant by adding the scores in the food categories (theoretically ranging from 0 to 9) with higher score indicating higher adherence to the MeDi.

Because ingestion of ethanol diminishes the amplitude of the tremor in ET [16, 17], it is conceivable that ET cases might modify their ethanol use for this reason. Because ethanol use is considered in the traditional calculation of the $\mathrm{MeDi}$ (as described above) we conducted supplementary analyses to address this issue. In initial models we adjusted for ethanol intake. In subsequent models, we recalculated the MeDi score without considering ethanol (range $0-8$ ) and reran the analyses (again simultaneously adjusting for ethanol intake).

\section{Covariates}

Weight and height were assessed using a balance scale designed for field surveys (Scale-Tronix 5600, White Plains, N.Y., USA) and a movable anthropometer (GPM Martin Type, Pfister Inc., Carlstadt, N.J., USA) and body mass index was calculated as weight in kilograms divided by the square of height in meters $\left(\mathrm{kg} / \mathrm{m}^{2}\right)$ [23] Age (years), education (years), caloric intake (kcal), and body mass index were used as continuous variables. Current cigarette smoking was assessed in each subject based on self-report: current smokers and nonsmokers (reference group). We also considered gender (men as reference) and ethnic group based on self-report using the format of the 1990 census with participants being assigned to one of four groups: Black (non-Hispanic), Hispanic, White (non-Hispanic) or Other. Ethnicity was used as a dummy variable with White (non-Hispanic) as the reference. We finally considered caffeine intake (milligrams; continuous variable) and blood harmane (logarithmically transformed because of non-normal distribution $[7,8])$ in the analyses. Details regarding harmane measurements have been described elsewhere $[7,8]$.

\section{Statistical Analyses}

Characteristics of subjects by outcome of interest (i.e. ET case vs. control status) and by MeDi tertiles were compared using $t$ test or ANOVA for continuous variables and $\chi^{2}$ test for categorical variables. We calculated logistic regression models with diagnostic status as the dichotomous outcome: ET versus controls. The main predictor was $\mathrm{MeDi}$ score as a continuous variable initially and in tertiles form subsequently (used for trend test calculation). In order to control for possible demographic and clinical differences among ET patients and controls, in subsequent models we simultaneously adjusted for the following variables: age, gender, ethnicity, education, caloric intake, body mass index, smoking, ethanol consumption, coffee intake and blood harmane concentrations. Our rationale for including the above variables in the model was as follows. Age, gender, ethnicity and education are basic demographic variables. Furthermore, ethnicity, education and smoking status can be considered as indirect indicators of socioeconomic status and health consciousness, factors often related to dietary choices. We included ethanol because, as explained earlier, it has a symptomatic effect on tremor [16, 17], and ET cases might modify their ethanol use for this reason. We considered caffeine and blood harmane because both previous work $[7,8,11]$ and current data demonstrate case-control differences.

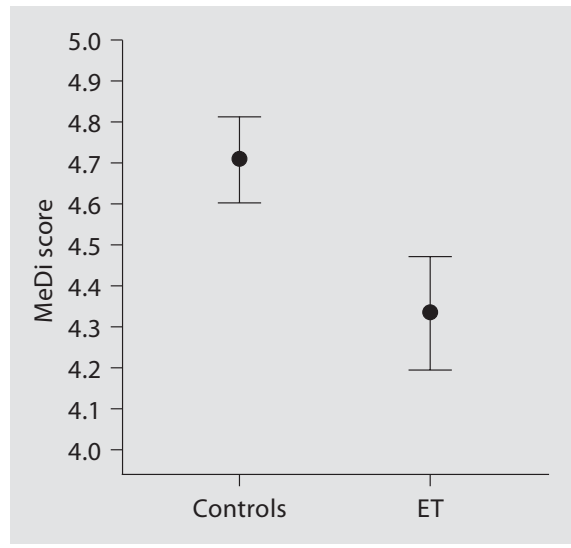

Fig. 1. Means and standard errors of $\mathrm{MeDi}$ score for ET patients and controls.

Because subjects' food intake is related to their body size, body mass index is commonly included in dietary analyses. Finally, although caloric intake-adjusted residuals were used in the MeDi score calculation, we also included caloric intake as a covariate in the models, as recommended by Willett and Stampfer [22].

\section{Results}

\section{Clinical, Demographic, Dietary Characteristics}

ET patients had the disease for an average of 23.7 $( \pm 18.7)$ years and $62 \%$ of them were taking medications for ET symptom control. Family history of tremor was more common for ET patients (65\%) as compared to controls $(35 \%$; $\mathrm{p}<0.001)$. Compared with controls, ET patients were, on average, 4.3 years older and in accordance to our previous reports [11], consumed less caffeine and had higher blood levels of harmane $[7,8]$ (table 1). Controls and ET patients did not differ in gender, ethnicity, education, smoking status, ethanol consumption, body mass index and caloric intake. Compared to controls, patients with ET had lower MeDi scores (table 1, fig. 1).

There was no association between MeDi score and gender, ethnicity, smoking status, harmane levels, and caloric intake (table 2). Subjects adhering more to the MeDi were older and had lower body mass index. There was a nonsignificant trend for higher MeDi adherence to be associated with higher education and lower caffeine consumption.

\section{MeDi and ET}

Higher adherence to the MeDi was associated with significantly lower odds of ET (table 3, fig. 2). The results 
Table 1. Demographic, clinical and dietary characteristics for ET cases and controls

\begin{tabular}{lcccc}
\hline & $\begin{array}{l}\text { ET } \\
(\mathrm{n}=148)\end{array}$ & $\begin{array}{c}\text { Controls } \\
(\mathrm{n}=250)\end{array}$ & $\begin{array}{l}\text { All } \\
(\mathrm{n}=398)\end{array}$ & $\mathrm{p}$ \\
\hline Age, years & $71.3 \pm 12.0$ & $67.0 \pm 11.6$ & $68.3 \pm 11.9$ & 0.001 \\
Male gender, $\mathrm{n}(\%)$ & $61(41)$ & $102(41)$ & $163(41)$ & 0.94 \\
Education, years & $14.6 \pm 3.4$ & $15.1 \pm 3.4$ & $14.9 \pm 3.4$ & 0.15 \\
Ethnicity, n (\%) & & & & 0.24 \\
$\quad$ White & $138(94)$ & $221(88)$ & $359(90)$ & \\
$\quad$ Black & $3(2)$ & $13(5)$ & $16(4)$ & \\
$\quad$ Hispanic & $5(3)$ & $10(4)$ & $15(4)$ & \\
$\quad$ Other & $1(1)$ & $6(2)$ & $7(2)$ & \\
Smoking, n $\%)$ & $9(8)$ & $26(11)$ & $35(10)$ & 0.35 \\
Caffeine, mg & $123 \pm 147$ & $228 \pm 209$ & $189 \pm 195$ & $<0.001$ \\
Ethanol, g & $6.9 \pm 12.7$ & $6.1 \pm 9.9$ & $6.4 \pm 11.0$ & 0.45 \\
Log harmane & $0.7 \pm 0.6$ & $0.5 \pm 0.7$ & $0.5 \pm 0.70$ & 0.040 \\
Body mass index & $28.0 \pm 12.7$ & $28.0 \pm 5.6$ & $27.9 \pm 8.7$ & 0.98 \\
Energy, kcal & $1,397 \pm 432$ & $1,421 \pm 487$ & $1,412 \pm 467$ & 0.63 \\
MeDi score & $4.3 \pm 1.7$ & $4.7 \pm 1.7$ & $4.6 \pm 1.7$ & 0.03 \\
MeDi tertiles, $\mathrm{n}(\%)$ & & & & 0.005 \\
$\quad$ Low & $56(38)$ & $57(23)$ & $113(28)$ & \\
Middle & $49(33)$ & $109(44)$ & $158(40)$ & \\
$\quad$ High & $43(29)$ & $84(34)$ & $127(32)$ & \\
\hline
\end{tabular}

Mean values \pm SD are given except for gender, ethnicity, smoking and MeDi tertiles. Italics: statistically significant.

Table 2. Demographic and clinical characteristics by MeDi score tertiles

\begin{tabular}{lcccc}
\hline & $\begin{array}{l}\text { Low tertile } \\
(\text { MeDi score 0-3) }\end{array}$ & $\begin{array}{l}\text { Middle tertile } \\
(\text { MeDi score 4-5) }\end{array}$ & $\begin{array}{l}\text { High tertile } \\
(\text { MeDi score 6-9) }\end{array}$ & $p$ \\
\hline Age, years & $65.7 \pm 13.0$ & $69.3 \pm 11.5$ & $70.5 \pm 11.0$ & $0.005^{\mathrm{a}}$ \\
Male gender, n (\%) & $41(36)$ & $63(40)$ & $59(47)$ & 0.26 \\
Education, years & $14.6 \pm 3.3$ & $14.8 \pm 3.4$ & $15.4 \pm 3.4$ & 0.13 \\
Ethnicity, n (\%) & $100(89)$ & $141(89)$ & $118(93)$ & 0.41 \\
$\quad$ White & $6(5)$ & $7(4)$ & $3(2)$ & \\
$\quad$ Black & $5(5)$ & $8(5)$ & $2(2)$ & 0.87 \\
$\quad$ Hispanic & $1(1)$ & $15(10)$ & $10(9)$ & 0.15 \\
$\quad$ Other & $10(10)$ & $201 \pm 204$ & $162 \pm 162$ & $0.03^{\mathrm{a}}$ \\
Smoking, n (\%) & $202 \pm 212$ & $0.4 \pm 0.7$ & $26.9 \pm 5.8$ & 0.58 \\
Caffeine, mg & $0.6 \pm 0.7$ & $27.6 \pm 5.0$ & $1,431 \pm 467$ & \\
Log harmane & $29.8 \pm 14.3$ & $1,425 \pm 428$ & & \\
Body mass index & $1,373 \pm 506$ & & & \\
Energy, kcal & & & & \\
\hline
\end{tabular}

Mean values \pm SD are given except for gender, ethnicity and smoking. Italics: statistically significant.

${ }^{a}$ Post-hoc Scheffé's tests for age: low vs. high tertile, $p=0.007$; low vs. middle, $p=0.05$; middle vs. high, $p=$ 0.69 ; for body mass index: low vs. high tertile, $\mathrm{p}=0.03$; low vs. middle, $\mathrm{p}=0.11$; middle vs. high, $\mathrm{p}=0.75$.

were similar in adjusted and unadjusted models. Each additional unit of the MeDi score was associated with a 12 $22 \%$ reduction in odds of ET. In unadjusted models, compared to subjects in the lowest MeDi tertile (low adher- ence to the $\mathrm{MeDi}$ ), subjects in the middle MeDi score tertile had $54 \%$ lower odds of ET, while those at the highest tertile (high adherence to the MeDi) had $48 \%$ lower odds of ET. Despite resulting in a considerable sample size 
Table 3. Odds ratios (ORs) for ET versus control status by MeDi score either in continuous or in tertile form

\begin{tabular}{|c|c|c|c|c|c|c|c|c|}
\hline Model & ET & Controls & $\begin{array}{l}\text { MeDi continuous OR } \\
(95 \% \mathrm{CI})\end{array}$ & $\mathrm{p}$ & $\begin{array}{l}\text { MeDi } \\
\text { tertiles }\end{array}$ & OR (95\% CI) & $\mathrm{p}$ & $\begin{array}{l}\mathrm{p} \\
\text { for trend }\end{array}$ \\
\hline 1 & 148 & 250 & $0.88(0.78-0.99)$ & 0.035 & $\begin{array}{l}\text { low } \\
\text { middle } \\
\text { high }\end{array}$ & $\begin{array}{l}1 \text { (reference) } \\
0.46(0.28-0.75) \\
0.52(0.31-0.88)\end{array}$ & $\begin{array}{l}0.002 \\
0.014\end{array}$ & 0.015 \\
\hline 2 & 52 & 140 & $0.78(0.61-0.99)$ & 0.042 & $\begin{array}{l}\text { low } \\
\text { middle } \\
\text { high }\end{array}$ & $\begin{array}{l}1 \text { (reference) } \\
0.41(0.16-1.05) \\
0.29(0.10-0.82)\end{array}$ & $\begin{array}{l}0.064 \\
0.020\end{array}$ & 0.021 \\
\hline
\end{tabular}

Model 1 is unadjusted. Model 2 is adjusted for age, gender, ethnicity, education, body mass index, caloric intake, smoking, caffeine, ethanol and blood harmane. Italics: statistically significant.

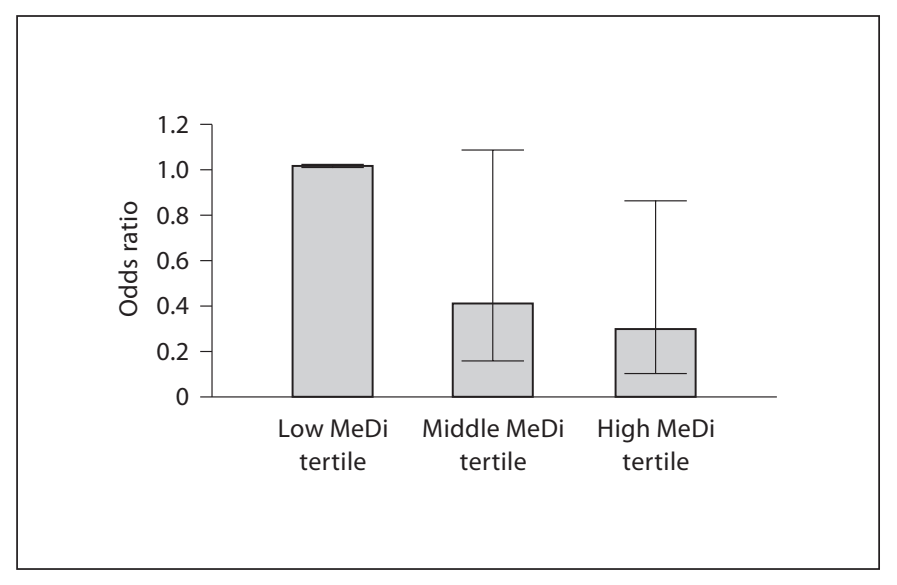

Fig. 2. Odds ratios and $95 \%$ confidence intervals (bars) for ET patients versus controls, for each $\mathrm{MeDi}$ adherence tertile, based on logistic regression models that adjusted for age, gender, ethnicity, education, body mass index, caloric intake, smoking, caffeine and blood harmane.

reduction, adjustment for all potential covariates made the associations even stronger: $59 \%$ reduction in odds for the middle and $71 \%$ reduction in odds for the highest $\mathrm{MeDi}$ tertile, with a significant trend for a dose-response effect.

When the same fully adjusted models were run with MeDi score calculated without considering ethanol, the associations were unchanged: MeDi continuous odds ratio $0.75(0.58-0.98), \mathrm{p}=0.034$; tertile analyses (lower tertile as reference), MeDi middle tertile 0.32 (0.11-0.93), MeDi highest tertile $0.30(0.12-0.76), \mathrm{p}$ for trend $=$ 0.011 .

\section{Discussion}

We observed that higher adherence to the $\mathrm{MeDi}$ is associated with reduced odds of ET. We noted a gradual reduction in the odds of ET for higher tertiles of $\mathrm{MeDi}$ adherence, suggesting a possible dose-response effect. Additionally, the associations between $\mathrm{MeDi}$ and ET remained unchanged and significant even when simultaneously adjusting for a multitude of potential confounding factors.

Although the etiology of ET is poorly understood [4] there is evidence that nongenetic (e.g., toxic or dietary) factors, in addition to the well-established genetic factors, may play an important role [4, 7-9]. There have been previous attempts to relate dietary factors with $\mathrm{ET}$ and the results have been variable. While blood levels of the neurotoxin harmane are elevated in ET cases, and harmane is found in the human diet in the animal proteins (meat), an early study indicated that animal protein consumption did not seem to differ between ET patients and controls [7]. As compared to controls, ET patients drink less coffee but consume similar amounts of ethanol, tea, soft drinks, fruit juices, and milk [11]. Finally, nutritional antioxidant (vitamins $\mathrm{E}$ and $\mathrm{C}$ ) consumption does not seem to be different between ET patients and controls [12].

We add to the literature the observation that ET patients may adhere less to an overall 'healthy' dietary pattern. However, the mechanism by which the MeDi may be protective for ET is far from clear. It has been suggested that one of the possible mechanisms for ET pathogenesis could be neurodegenerative [13-15]. Vascular [2527], oxidative [28, 29] and inflammatory [24, 27, 30-33] biological mechanisms have been implicated in neurodegeneration and the MeDi has been associated with all 
three above pathways. There is strong evidence relating the $\mathrm{MeDi}$ to lower risk of a series of vascular risk factors including dyslipidemia [34, 35], hypertension [34-37], abnormal glucose metabolism $[35,37]$ and coronary heart disease $[21,35,38,39]$. Food intervention studies with typical MeDi elements have indicated significant reductions of markers of oxidative stress [40,41]. Consumption of many nutritional antioxidants did not differ between ET patients and controls according to a previous study [12], but the MeDi, being a composite dietary pattern, may be better at capturing the overall antioxidant effect of the diet. Higher adherence to the MeDi has been associated with lower levels of a series of markers of inflammation in both observational $[34,42,43]$ and interventional $[37,44]$ studies.

It is possible that lower adherence to the $\mathrm{MeDi}$ could represent a consequence and not a cause of ET because ET patients may increase ethanol intake in an attempt to improve their symptoms [16, 17]. Although we cannot completely exclude this, we addressed this consideration in multiple ways. First, we detected no difference in ethanol consumption per se between ET cases and controls. Second, we adjusted for ethanol in our analyses and the associations between MeDi and ET persisted. Third, we observed a significant association even when we recalculated the MeDi without considering ethanol.

Given the cross-sectional design of this study, it is possible that lower adherence to the MeDi could represent a consequence and not a cause of ET. Therefore, longitudinal studies of incident cases are needed. In two previous publications, however, using a subset of subjects with repeated (2-4) dietary assessments over a course of $\sim 8$ (and up to 13) years, we demonstrated that adherence to the $\mathrm{MeDi}$ is remarkably stable over time $[2,3]$. Therefore, it is conceivable that the $\mathrm{MeDi}$ adherence reported for our ET population could reflect their long-standing (predisease) dietary habits.

This study has limitations. First, the use of an a priori distribution-derived $\mathrm{MeDi}$ score assumes underlying monotonic effects, does not address possible thresholds or the shape of the underlying curve and weighs equally the underlying individual food categories, which in turn are composed of different number of food constituents. Frequencies of food intake are based on relatively few diet constituents, which may underestimate the overall quantity of food in each food category and a common limitation of studies of diet and disease is misclassification of exposure due to limited accuracy. However, assuming that the measurement error was random, our results may actually underestimate the association between high
MeDi adherence and odds of ET. Second, because the vast majority of our subjects were clinic-based and of white ethnicity, our sample is not representative of the population, which may limit the external validity - generalizability of our findings. Third, as compared to subjects included in the present analyses, excluded subjects were slightly more educated and younger. Education was statistically unrelated to both our predictor and our outcome, but older subjects adhered more to the MeDi and ET patients were 4 years older than controls. We attempted to address this by including both education and age as potential confounders in our analyses: not only did the associations not attenuate but they became even stronger. Therefore, although we cannot completely exclude it, it does not seem likely that our results could be explained by biases related to missing dietary information. Fourth, it is possible that diet is related to socioeconomic status or to other habits or characteristics related to better health and a lower risk for ET. In our sample, MeDi was not related to education, smoking habits or ethnicity and we further addressed this potential bias by adjusting for all the above. In additional analyses (not shown) we also considered other indices of socioeconomic status such as number of people living with the subjects, occupational attainment and an index of other medical comorbidities, but none of these differed between ET patients and controls. Still, we cannot completely rule out residual confounding as an explanation for our findings.

Confidence in our findings is strengthened by the following factors. Dietary data were collected with a previously validated and widely used instrument [20]. We used an a priori developed dietary pattern [21]. Measures for multiple potential confounding factors have been carefully recorded and adjusted for in the analyses. According to expectations, $\mathrm{MeDi}$ adherence was higher in older and (possibly) in more educated persons, while subjects adhering more to the MeDi had lower body mass index and (possibly) consumed less caffeine; these findings add to the validity of the $\mathrm{MeDi}$ assessments in our population. The diagnosis of ET took place in a University hospital with expertise in this condition and was based on comprehensive assessment and standard research criteria.

In conclusion, our results indicate that the recently reported beneficial effects of MeDi for Alzheimer's disease $[2,3]$ may extend to ET, a very common neurological disease without known prevention or effective treatment [5, $6,45]$. It has been argued that it is easier to prescribe drugs than to change the dietary habit of subjects. However, the Lyon Diet Heart study indicated that most subjects were 
still following the MeDi several years after randomization [38]. The American Heart Association has issued a scientific advisory stating that a Mediterranean-style diet demonstrates impressive effects on cardiovascular dis- ease [46]. However, it is very premature to recommend the MeDi for prevention of ET based on our observational study, which is only the first report of such an association and should be replicated.

\section{References}

1 Jacques PF, Tucker KL: Are dietary patterns useful for understanding the role of diet in chronic disease? Am J Clin Nutr 2001;73:12.

-2 Scarmeas N, Stern Y, Mayeux R, Luchsinger JA: Mediterranean diet, Alzheimer disease, and vascular mediation. Arch Neurol 2006; 63:1709-1717.

3 Scarmeas N, Stern Y, Tang MX, Mayeux R, Luchsinger JA: Mediterranean diet and risk for Alzheimer's disease. Ann Neurol 2006; 59:912-921.

$\checkmark 4$ Louis ED: Etiology of essential tremor: should we be searching for environmental causes? Mov Disord 2001;16:822-829.

$\checkmark 5$ Benito-Leon J, Bermejo-Pareja F, Louis ED: Incidence of essential tremor in three elderly populations of central Spain. Neurology 2005;64:1721-1725.

6 Dogu O, Sevim S, Camdeviren H, et al: Prevalence of essential tremor: door-to-door neurologic exams in Mersin Province, Turkey. Neurology 2003;61:1804-1806.

7 Louis ED, Zheng W, Applegate L, Shi L, Factor-Litvak P: Blood harmane concentrations and dietary protein consumption in essential tremor. Neurology 2005;65:391-396.

$\checkmark 8$ Louis ED, Zheng W, Jurewicz EC, et al: Elevation of blood beta-carboline alkaloids in essential tremor. Neurology 2002;59:19401944.

$\checkmark 9$ Louis ED, Jurewicz EC, Applegate L, et al: Association between essential tremor and blood lead concentration. Environ Health Perspect 2003;111:1707-1711.

-10 Louis ED, Applegate LM, Factor-Litvak P, Parides MK, Andrews L: Essential tremor: occupational exposures to manganese and organic solvents. Neurology 2004;63:21622164.

-11 Louis ED, Jurewicz EC, Applegate L, Luchsinger JA, Factor-Litvak P, Parides M: Semiquantitative study of current coffee, caffeine, and ethanol intake in essential tremor cases and controls. Mov Disord 2004;19:499-504.

12 Louis ED, Jurewicz EC, Parides MK: Casecontrol study of nutritional antioxidant intake in essential tremor. Neuroepidemiology 2005;24:203-208.

13 Louis ED, Jurewicz EC, Watner D: Community-based data on associations of disease duration and age with severity of essential tremor: implications for disease pathophysiology. Mov Disord 2003;18:90-93.
14 Louis ED, Shungu DC, Chan S, Mao X, Jurewicz EC, Watner D: Metabolic abnormality in the cerebellum in patients with essential tremor: a proton magnetic resonance spectroscopic imaging study. Neurosci Lett 2002; 333:17-20.

15 Louis ED, Vonsattel JP, Honig LS, Ross GW, Lyons KE, Pahwa R: Neuropathologic findings in essential tremor. Neurology 2006;66: 1756-1759.

16 Growdon JH, Shahani BT, Young RR: The effect of alcohol on essential tremor. Neurology 1975;25:259-262.

17 Koller WC, Biary N: Effect of alcohol on tremors: comparison with propranolol. Neurology 1984;34:221-222.

18 Louis ED, Ottman R, Ford B, et al: The Washington Heights-Inwood Genetic Study of Essential Tremor: methodologic issues in essential-tremor research. Neuroepidemiology 1997; 16:124-133.

19 Louis ED, Ford B, Lee H, Andrews H: Does a screening questionnaire for essential tremor agree with the physician's examination? Neurology 1998;50:1351-1357.

20 Willett WC, Sampson L, Stampfer MJ, et al: Reproducibility and validity of a semiquantitative food frequency questionnaire. Am J Epidemiol 1985;122:51-65.

21 Trichopoulou A, Costacou T, Bamia C, Trichopoulos D: Adherence to a Mediterranean diet and survival in a Greek population. N Engl J Med 2003;348:2599-2608.

22 Willett W, Stampfer M: Implications of total energy intake for epidemiological analyses; in Willett W (ed): Nutritional Epidemiology. New York, Oxford University Press, 1998, pp 273-301.

23 Kuczmarski R, Carroll M, Flegal K, Troiano $\mathrm{R}$ : Varying body mass index cutoff points to describe overweight prevalence among U.S. adults: NHANES III (1988 to 1994). Obes Res 1997;5:542-548.

24 Sastre M, Klockgether T, Heneka MT: Contribution of inflammatory processes to Alzheimer's disease: molecular mechanisms. Int J Dev Neurosci 2006;24:167-176.

25 Breteler MM: Vascular risk factors for Alzheimer's disease: an epidemiologic perspective. Neurobiol Aging 2000;21:153-160.

26 Luchsinger J, Mayeux R: Cardiovascular risk factors and Alzheimer's disease. Curr Atheroscler Rep 2004;6:261-266.

27 Cummings JL: Alzheimer's disease. N Engl J Med 2004;351:56-67.
28 Giasson BI, Ischiropoulos H, Lee VM, Trojanowski JQ: The relationship between oxidative/nitrative stress and pathological inclusions in Alzheimer's and Parkinson's diseases. Free Radic Biol Med 2002;32:12641275.

29 Pratico D: Alzheimer's disease and oxygen radicals: new insights. Biochem Pharmacol 2002;63:563-567.

-30 Walsh S, Aisen PS: Inflammatory processes and Alzheimer's disease. Expert Rev Neurother 2004;4:793-798.

31 Weninger SC, Yankner BA: Inflammation and Alzheimer disease: the good, the bad, and the ugly. Nat Med 2001;7:527-528.

32 Akiyama H, Barger S, Barnum S, et al: Inflammation and Alzheimer's disease. Neurobiol Aging 2000;21:383-421.

33 Yaffe K, Kanaya A, Lindquist K, et al: The metabolic syndrome, inflammation, and risk of cognitive decline. JAMA 2004;292: 2237-2242.

34 Chrysohoou C, Panagiotakos DB, Pitsavos C, Das UN, Stefanadis C: Adherence to the Mediterranean diet attenuates inflammation and coagulation process in healthy adults: The ATTICA Study. J Am Coll Cardiol 2004;44:152-158.

35 Singh RB, Dubnov G, Niaz MA, et al: Effect of an Indo-Mediterranean diet on progression of coronary artery disease in high risk patients (Indo-Mediterranean Diet Heart Study): a randomised single-blind trial. Lancet 2002;360:1455-1461.

36 Psaltopoulou T, Naska A, Orfanos P, Trichopoulos D, Mountokalakis T, Trichopoulou A: Olive oil, the Mediterranean diet, and arterial blood pressure: the Greek European Prospective Investigation into Cancer and Nutrition (EPIC) study. Am J Clin Nutr 2004;80:1012-1018

37 Esposito K, Marfella R, Ciotola M, et al: Effect of a Mediterranean-style diet on endothelial dysfunction and markers of vascular inflammation in the metabolic syndrome: a randomized trial. JAMA 2004;292:14401446.

38 de Lorgeril M, Salen P, Martin JL, Monjaud I, Delaye J, Mamelle N: Mediterranean diet, traditional risk factors, and the rate of cardiovascular complications after myocardial infarction: final report of the Lyon Diet Heart Study. Circulation 1999;99:779-785. 
39 Knoops KT, de Groot LC, Kromhout D, et al: Mediterranean diet, lifestyle factors, and 10 year mortality in elderly European men and women: the HALE project. JAMA 2004;292: 1433-1439.

40 Mancini M, Parfitt VJ, Rubba P: Antioxidants in the Mediterranean diet. Can J Cardiol 1995;11(suppl G):105G-109G.

-41 Sanchez-Moreno C, Cano MP, de Ancos B, et al: Mediterranean vegetable soup consumption increases plasma vitamin $\mathrm{C}$ and decreases F2-isoprostanes, prostaglandin E2 and monocyte chemotactic protein-1 in healthy humans. J Nutr Biochem 2006;17: 183-189.
2 Paschos GK, Rallidis LS, Liakos GK, et al: Background diet influences the anti-inflammatory effect of alpha-linolenic acid in dyslipidaemic subjects. Br J Nutr 2004;92:649655.

43 Fung TT, McCullough ML, Newby P, et al: Diet-quality scores and plasma concentrations of markers of inflammation and endothelial dysfunction. Am J Clin Nutr 2005;82: 163-173.
44 Blum S, Aviram M, Ben-Amotz A, Levy Y: Effect of a Mediterranean meal on postprandial carotenoids, paraoxonase activity and C-reactive protein levels. Ann Nutr Metab 2006;50:20-24.

45 Louis ED: Clinical practice: essential tremor. N Engl J Med 2001;345:887-891.

46 Kris-Etherton P, Daniels SR, Eckel RH, et al: Summary of the scientific conference on dietary fatty acids and cardiovascular health: conference summary from the Nutrition Committee of the American Heart Association. Circulation 2001;103:1034-1039. 